\section{(2) OPEN ACCESS}

\title{
Human leucocyte antigen alleles confer susceptibility and progression to Graves' ophthalmopathy in a Southern Chinese population
}

\author{
Xiaosheng Huang (10 1,2 Guiqin Liu, ${ }^{1,2}$ Shaoyi Mei, ${ }^{1,2}$ Jiamin Cai, ${ }^{2}$ Jing Rao, ${ }^{1}$ \\ Minzhong Tang, ${ }^{3}$ Tianhui Zhu, ${ }^{1,2}$ Wenchiew Chen, ${ }^{1}$ Shiming Peng, ${ }^{1,2}$ Yan Wang, ${ }^{1}$ \\ Ye Ye, ${ }^{2}$ Tong Zhang, ${ }^{1}$ Zhihui Deng (10, ${ }^{4,5}$ Jun Zhao (1) ${ }^{1,2}$
}

For numbered affiliations see end of article.

\section{Correspondence to}

Zhihui Deng, Immunogenetics Laboratory, Shenzhen Blood

Center, Shenzhen, China, and Department of Transfusion Medicine, School of Laboratory Medicine and Biotechnology, Southern Medical University, Guangzhou, China; zhihui_deng@aliyun.com Jun Zhao, Shenzhen Eye Institute, Shenzhen Eye Hospital Affiliated to Jinan University and School of Ophthalmology \& Optometry Affiliated to Shenzhen University, Shenzen, China; doctorzhaojun@163.com

XH and GL contributed equally ZD and IZ contributed equally

Received 1 June 2020

Revised 12 October 2020

Accepted 26 October 2020

\section{Check for updates}

(C) Author(s) (or their employer(s)) 2020. Re-use permitted under CC BY-NC. No commercial re-use. See rights and permissions. Published by BMJ.

To cite: Huang $X$, Liu $G$, Mei $S$, et al. Br J

Ophthalmol Epub ahead of print: [please include Day Month Year]. doi:10.1136/ bjophthalmol-2020-

317091

\begin{abstract}
Purpose To evaluate the contributions of human leucocyte antigen (HLA) class I and II genes in the development of Graves' ophthalmopathy (GO) in a Southern Chinese population.
\end{abstract}

Methods Eight HLA loci were genotyped and analysed in 272 unrelated patients with Graves' disease (GD) or the proptosis and myogenic phenotypes of GO, and 411 ethnically matched control subjects.

Results The allele frequencies of $H L A-D R B 1{ }^{*} 16: 02$ and $-D Q B 1{ }^{*} 05: 02$ in the GD, proptosis and myogenic groups, $H L A-B * 38: 02$ and $-D Q A 1 * 01: 02$ in the myogenic group were significantly higher than those in the control group, respectively (all corrected $p$ values $<0.05, O R>2.5$ ). The haplotype frequencies of $H L A-D R B 1 * 16: 02-D Q A 1 * 01$ $: 02-D Q B 1{ }^{*} 05: 02$ and HLA-DRB1* 16:02-DQA1*01:02$D Q B 1{ }^{*} 05: 02-D P A 1{ }^{*} 02: 02-D P B 1{ }^{*} 05: 01$ in the proptosis and myogenic groups, and $H L A-A{ }^{*} 02: 03-B * 38: 02-C * 07$ $: 02$ and $H L A-A{ }^{*} 02: 03-B{ }^{*} 38: 02-C{ }^{*} 07: 02-D R B 1 * 16: 02-$ $D Q A 1$ *01:02-DQB 1 *05:02-DPA1 *02:02-DPB1 *05:01 in the myogenic group were significantly higher than those in the control group respectively (all corrected $p$ values $<0.05, O R>2.5$ ). The potential epitopes ('FLGIFNTGL' of TSHR, 'IRHSHALVS', 'ILYIRTNAS' and 'FVFARTMPA' of IGF-1R) were fitted exactly in the peptide-binding groove between HLA-DRA1-DRB1*16 :02 heterodimer, and the epitopes ('ILEITDNPY' of THSR, 'NYALVIFEM' and 'NYSFYVLDN' of IGF-1R) were also fitted exactly in the peptide-binding groove between $H L A$ $D Q A 1 * 01: 02-D Q B 1 * 05: 02$ heterodimer.

Conclusions The HLA-DRB1*16:02 and $-D Q B 1{ }^{*} 01: 02$ alleles might be risk factors for $G D$ including the proptosis and myogenic phenotypes of G0. The alleles $H L A-B{ }^{*} 38: 02$, $-D Q A 1{ }^{*} 01: 02$, the HLA haplotypes consisting of $H L A-B * 38$ :02, $-D R B 1 * 16: 02,-D Q A 1 * 01: 02$ and $-D Q B 1{ }^{*} 05: 02$ might be susceptibility risk factors for $\mathrm{GO}$. Simultaneously, some epitopes of TSHR and IGF-1R tightly binding to groove of HLA-DRA1-DRB1 *16:02 or HLA-DQA1*01:02-DQB1 *05 $: 02$ heterodimers might provide some hints on presenting the pathological antigen in $\mathrm{GO}$.

\section{INTRODUCTION}

Graves' ophthalmopathy (GO) is a common autoimmune inflammatory eye disease of adulthood associated with Graves' disease (GD), whose predisposition is widely attributed to interplay of genetic and environmental factors. ${ }^{1}$ The common symptoms of GO are dry, photophobia, double vision and pressure sensation behind the eyes. ${ }^{1}$ The clinical phenotypes of $\mathrm{GO}$ are proptosis and restrictive extraocular myopathy. ${ }^{2}$ Over the years, genomewide association studies have identified a growing list of credible candidate human leucocyte antigen (HLA) loci modestly associated with GD. ${ }^{3}{ }^{4}$ The main function of HLA molecules is to present antigenic peptides to the immune system and thus regulate the immune response. ${ }^{5}$ The highly polymorphic HLA system is one of key immunogenetics associated with GO. ${ }^{6}$ The genetic variations of classical class I region (eg, HLA-B) and the class II region (eg, HLA$D R B 1,-D Q 1$, and $-D P B 1)$ are risk or protective factors in some ethnic populations, resulting in differences with GO prevalence. $^{6}$ In the Brazilian population, $H L A-D R B 1 * 16$ was over-represented in myogenic subtype of GO patients, and HLA$D R B 1 * 03$ was over-represented in non-myogenic subtype of GO patients. ${ }^{7}$ In the Polish population, the HLA-DRB1*03 allele was found to be positively associated with GO. ${ }^{8}$ Previous studies have shown that low-resolution HLA genotypes and serological HLA antigens are associated with GO. In the Japanese population, the antigens (HLA-DR14 and DQ1) might be the genetic markers of predisposition to GO. ${ }^{9}$ In the British population, the antigens (HLAB8, -DQw 3.1 and -DPB 2.1/8) might confer protective effects in patients with GO. ${ }^{10} 11$ Associations of $H L A-B 8$ and $-D R 3$ genotypes with GO were also reported in the Hungarian population. ${ }^{12}$

Although the initiating trigger of GO is still unclear, the excessive inflammation responses in orbital soft tissue might be the key pathogenesis. ${ }^{1} 13$ Thyroid stimulating hormone receptor (TSHR) and type 1 insulin-like growth factor receptor (IGF-1R) might be two major antigens in orbital microenvironment with GO. ${ }^{14} 15$ The extracellular domain of TSHR (TSHR-ECD) peptides epitopes bind to the groove of HLA molecules, forming HLA-THSRcomplexes, which are presented to $\mathrm{CD} 4^{+} \mathrm{T}$ cells in Graves' disease (GD). ${ }^{13}{ }^{15-18}$ Krieger et al have reported that bidirectional TSHR/IGF-1R crosstalk can mediate GO pathogenesis. ${ }^{19}$ Mahdavi et al found that some peptides of IGF-1R-ECD (86 and 249 epitopes) were the best HLA-binding peptide epitopes to both HLA class I and II molecules based on molecular docking analyses. ${ }^{20}$ As a result, activated orbital fibroblasts and infiltrating lymphocytes might contribute to the pathological phenotype of GO by producing 
glycosaminoglycan and differentiate into myofibroblasts or adipocytes. ${ }^{1} 13$

In the present study, we comprehensively conducted highresolution genotyping for eight HLA loci including $H L A-A$, $-B$, $-C,-D R B 1,-D Q A 1,-D Q B 1,-D P A 1$ and $-D P B 1$ in samples collected from 272 unrelated GD patient with and without clinical GO and 411 control subjects with high resolution at both allelic and haplotypic levels using PCR-sequence-based typing (SBT). We aimed to evaluate whether specific alleles, haplotypes and the dock condition of the THSR-ECD or IGF-1R-ECD segment in eight HLA loci contribute to the development of GO in Southern Chinese.

\section{MATERIALS AND METHODS Participants}

A total of 272 unrelated patients with GD were recruited from the patients attending the Clinic of Shenzhen Eye Hospital, China between December 2018 and December 2019. Diagnosis of GD was based on the typical clinical features of hyperthyroidism, diffuse goitre, suppressed TSHR levels, detectable THSR autoantibodies and/or increased radioiodine uptake. ${ }^{389}$ The clinical GO was defined as class 3 or higher in the American Thyroid Association mnemonic NOSPECS scheme. The GO patients divided into proptosis (the proptosis $\geq 18 \mathrm{~mm}$ or the difference between two eyes $\geq 2 \mathrm{~mm}$ ) and the myogenic (strabismus caused by extraocular-muscle dysfunction without orbital fat compartment enlargement) groups according to the presence of clinically evident ophthalmopathy, as previously described. ${ }^{2}{ }^{8} \mathrm{GD}$ patients without GO were selected from those who did not have any features of ophthalmopathy (including features of class 1-2). The control group consisted of 411 unrelated healthy volunteers randomly chosen from the Shenzhen Blood Centre, China. All patients and controls were Han Chinese from Southern China. The exclusion criteria were setting as follows: the heathy volunteer without autoimmunity systemic disease history including GDor GO, and the patient who was diagnosed as GD or GO without other systemicdiseases were enrolled as the study cohort. Peripheral blood samples (with 5\% EDTA as anti-coagulant) were collected from each subject and stored at $-20^{\circ} \mathrm{C}$. The study protocol was approved by the Ethics Committee of Shenzhen Eye Hospital and was in accordance with the tenets of the Declaration of Helsinki and its later amendments or comparable ethical standards. Written informed consent was obtained from all study participants.

\section{Subgroups of participants}

On the basis of the presence of clinically evident ophthalmopathy, four subgroups were considered: Group 1: GD without clinical eye phenotype group (no changes in ophthalmologic findings had been detected according to 3 years follow-up data); Group 2: the proptosis group (the proptosis $\geq 18 \mathrm{~mm}$ or the difference between two eyes $\geq 2 \mathrm{~mm}$ ); Group 3: the myogenic group (strabismus caused by extraocular-muscle dysfunction without orbital fat compartment enlargement); Group 4: the control group.

\section{DNA extraction and sequence-based typing for HLA genes}

Blood DNAs were obtained from all 683 participants. The IPDIMGT/HLA Database (http://www.ebi.ac.uk/ipd/imgt/hla/, Release 3.40.0, 2020 April) was applied for the genotyping of each locus. Genotyping for HLA-A, $-B,-C,-D R B 1$ and $-D Q B 1$ was performed using the AlleleSEQR HLA SBT commercial kit (Atria Genetics, San Francisco) according to the manufacturer's instructions. Exons $2 \sim 4$ for HLA-A, $-B,-C$ and exons 2, 3 for $-D R B 1$ and $-D Q B 1$ were sequenced. HLA-DQA1 SBT covered exons 1 4 as described by Voorter et al. ${ }^{21}$ HLA-DPA1 and -DPB1 SBT was performed according to the protocol developed by the 13 th International Histocompatibility Workshop. PCR amplification was performed using a GeneAmp PCR system 9700 (Applied Biosystems, Foster City, CA, USA). PCR products were purified using ExoSAP-IT (Atria Genetics) and sequenced with the ABI PRISM BigDye Terminator Cycle Sequencing Ready Reaction kits (Applied Biosystems, Foster City, CA, USA) on an ABI 3730XL DNA sequencer (Applied Biosystems, Foster City, CA, USA). Four-digit HLA genotypes were assigned with the help of the ASSIGN 3.5 software (Conexio Genomics, Applecross, Australia). Ambiguous allele combinations from the SBT results were further examined using the HLA PCR-SSP kit (Olerup, Stockholm, Sweden).

\section{HLA peptide-binding prediction and in silico docking}

The sequences of TSHR and IGF-1R (NP 000360.1 and NP 000866.1) were submitted to the NetMHCIIpan 3.2 server for predicting major HLA-II peptide-binding affinities. ${ }^{22}$ HLADRA1-DRB1*16:02 and HLA-DQA1*01:02-DQB1*05:02 heterodimers were selected for predicting epitopes within the protein. Threshold for weak and strong binding peptides was set as $1 \%$ and $10 \%$ ranks, respectively. The computing docking of the TSHR-ECD and IGF-1R-ECD segment into HLA-DRA1-DRB1*16:02 and HLADQA1*01:02-DQB1*05:02 heterodimers in silico was performed using the AutoDock Vina software, as described by Shu et al. ${ }^{23}$

\section{Statistical analysis}

Statistical analysis was performed using SPSS (version 20.0, SPSS Inc., Chicago, IL). Hardy-Weinberg equilibrium (HWE) was tested using the $\chi^{2}$ test. Haplotype frequencies were estimated from the genotyping results by the Excoffier-Laval-Balding (ELB) algorithm using the Arlequin software package version 3.5 (Laurent Excoffier, CMPG, Zoological Institute, University of Bern, Switzerland). ${ }^{24}$ The difference in allele frequency and haplotype frequency was tested using the $\chi^{2}$ test or Fisher's exact test. Linkage disequilibrium (LD), defined as D' and $r^{2}$, was calculated between each intra-gene haplotype block as described by Lewontin et al. ${ }^{25}$ Multiple testings were corrected using the Bonferroni method and the corrected $\mathrm{p}$ value was calculated by multiplying the $\mathrm{p}$ value with the number of tests performed. The corrected $\mathrm{p}$ values $(\mathrm{Pc})<0.05$ were considered statistically significant. OR and 95\% CI were calculated whenever applicable.

\section{RESULTS}

\section{Demographic and clinical features of participants}

In the GD without clinical eye phenotype group, there were 17 $(23.0 \%)$ males and $57(77.0 \%)$ females $(n=74)$, with an average age of $38.7( \pm 10.4)$ years. In the proptosis group $(n=82)$, there were $32(39.0 \%)$ males and $50(61.0 \%)$ females, with an average age of $34.9( \pm 12.1)$ years. In the myogenic group $(n=116)$, there were 59 $(51.0 \%)$ males and $57(49.1 \%)$ females, with an average age of 44.2 $( \pm 11.4)$ years. In the control group $(n=411)$, there were 411 healthy adults in Southern Chinese, with an average age of 40.6 $( \pm 12.0)$ years. No significant difference in age was found between patients with GD and controls $(39.9 \pm 12.0$ vs. $40.6 \pm 12.0$ years, $p$ value $=0.414)$.

\section{Allele frequencies of $H L A-A,-B,-C,-D R B 1,-D Q A 1,-D Q B 1$ and -DPB1}

The genotype distributions of HLA-A, -B, -C, -DRB1, -DQA1, $D Q B 1$ and $-D P B 1$ in the control group was in accordance with HWE (all p values $>0.05$ ) (data not shown). A total of $31 \mathrm{HLA}-\mathrm{A}$ alleles, 63 HLA-B alleles, 29 HLA-C alleles, 40 HLA-DRB1 alleles, 18 HLA-DQA1 alleles, 17 HLA-DQB1 alleles, 7 HLA-DPA1 
alleles, and 24 HLA-DPB1 alleles were identified in all participants including patients and controls (data not shown). The allele frequencies of HLA-DRB1*16:02 and -DQB1*05:02 in GD patients without clinical eye phenotype, the proptosis and myogenic groups were significantly higher than those in the control group respectively, which survived the Bonferroni correction (all Pc $<0.05$, OR $>2.5$ ). The allele frequencies of HLA-B*38:02 and -DQA1*01:02 in the myogenic group were significantly higher than those in the control group, respectively, which survived the Bonferroni correction $(8.6 \%$ vs $3.0 \%, \mathrm{Pc}=0.01, \mathrm{OR}=3.0 ; 32.8 \%$ vs $\left.19.8 \%, \mathrm{Pc}=5.9 \times 10^{-4}, \mathrm{OR}=2.0\right)($ table 1$)$.

\section{Haplotype frequencies}

We identified 404 HLA class I haplotypes (A-C-B), 93 HLADRB1-DQA1-DQB1 haplotypes, 44 HLA-DPA1-DPB1 haplotypes, 338 HLA class II haplotypes (HLA-DRB1-DQA1-DQB1DPA1-DPB1) and 974 HLA eight loci haplotypes (HLA A$C-B-D R B 1-D Q A 1-D Q B 1-D P A 1-D P B 1)$ in all participants including patients and controls (data not shown). The haplotype frequencies of HLA-DRB1*16:02-DQA1*01:02-DQB1*05:02 and HLA-DRB1*16:02-DQA1*01:02-DQB1*05:02-DPA1*02 $: 02-D P B 1 * 05: 01$ in the proptosis and myogenic groups were significantly higher than those in the control group, respectively, which survived the Bonferroni correction (all Pc $<0.05$, OR > 4.0). The haplotype frequencies of $H L A-A * 02: 03-B * 38$ :02-C*07:02 and HLA-A*02:03-B*38:02-C*07:02-DRB1*16 :02-DQA1*01:02-DQB1*05:02-DPA1*02:02-DPB1*05:01

in the myogenic group were significantly higher frequencies than those in the control group, respectively, which survived the Bonferroni correction (6.5 vs $2.7 \%, \mathrm{Pc}=0.02, \mathrm{OR}=4.3$; 4.7 vs $\left.0.4 \%, \mathrm{Pc}=1.7 \times 10^{-3}, \mathrm{OR}=13.6\right)($ table 1$)$.

Since the three disease-associated HLA class II alleles HLADRB1*16:02, HLA-DQA1*01:02 and HLA-DQB1*05:02 formed a haplotype, we further investigated the associations between eight combinations of HLA-DRB1*16:02, HLA$D Q A 1 * 01: 02$ and HLA-DQB1*05:02 with disease groups. As shown in table 2, HLA-DRB1*16:02 ${ }^{+}-D Q A 1 * 01: 02^{+}$. $D Q B 1 * 05: 02^{+}$was associated with the proptosis and myogenic groups, respectively, which survived the Bonferroni correction (all $\mathrm{Pc}<0.05, \mathrm{OR}=3.96$ and 4.83 respectively). HLA-DRB1*16:

Table 1 Allele and haplotype frequencies of HLA in cases and controls

\begin{tabular}{|c|c|c|c|c|c|c|c|c|c|c|}
\hline & \multirow{2}{*}{$\frac{\text { Controls }}{(2 \mathrm{n}=822, \%)}$} & \multicolumn{3}{|c|}{$\begin{array}{l}\text { GD without clinical eye } \\
\text { phenotype group }\end{array}$} & \multicolumn{3}{|c|}{ Proptosis group } & \multicolumn{3}{|c|}{ Myogenic group } \\
\hline & & $(2 \mathrm{n}=148, \%)$ & $\mathrm{Pc}^{*}$ & OR & $(2 \mathrm{n}=164, \%)$ & Pct & OR & $(2 \mathrm{n}=232, \%)$ & Pc‡ & OR \\
\hline \multicolumn{11}{|l|}{ HLA Allele } \\
\hline$B * 38: 02$ & $25(3.0)$ & $4(2.7)$ & NS & & $7(4.3)$ & NS & & $20(8.6)$ & 0.01 & 3.0 \\
\hline $\mathrm{DRB} 1 * 16: 02$ & $39(4.7)$ & $17(11.5)$ & 0.048 & 2.6 & 27 (16.5) & $1.7 \times 10^{-6}$ & 4.0 & 45 (19.4) & $1.4 \times 10^{-11}$ & 4.8 \\
\hline DQA1*01:02 & $163(19.8)$ & $38(25.7)$ & NS & & $42(25.6)$ & NS & & $76(32.8)$ & $5.9 \times 10^{-4}$ & 2.0 \\
\hline $\mathrm{DQB1}{ }^{*} 05: 02$ & $21(10.9)$ & 29 (19.6) & $1.1 \times 10^{-16}$ & 9.3 & $36(22.0)$ & $4.6 \times 10^{-21}$ & 10.7 & $64(27.6)$ & $7.2 \times 10^{-34}$ & 14.5 \\
\hline \multicolumn{11}{|l|}{ HLA Haplotype } \\
\hline$A * 02: 03-B * 38: 02-C * 07: 02$ & $13(1.6)$ & $4(2.7)$ & NS & & $0(0.0)$ & NS & & $15(6.5)$ & 0.02 & 4.3 \\
\hline DRB1 *16:02-DQA1 *01:02-DQB1 *05:02 & $39(4.7)$ & $17(11.5)$ & NS & & $27(16.5)$ & $3.9 \times 10^{-6}$ & 4.0 & $45(19.4)$ & $3.2 \times 10^{-11}$ & 4.8 \\
\hline $\begin{array}{l}\text { DRB1 * 16:02-DQA1 *01:02-DQB1 *05:02-D } \\
\text { PA1 *02:02-DPB1 *05:01 }\end{array}$ & $23(2.8)$ & $8(5.4)$ & NS & & $17(10.3)$ & $2 \times 10^{-3}$ & 4.0 & $37(15.9)$ & $7.7 \times 10^{-12}$ & 6.6 \\
\hline $\begin{array}{l}A^{*} 02: 03-B * 38: 02-C * 07: 02-D R B 1 * 16: 02-D Q A 1 * 01: \\
02-D Q B 1 * 05: 02-D P A 1 * 02: 02-D P B 1 * 05: 01\end{array}$ & $3(0.4)$ & $2(1.6)$ & NS & & $1(0.1)$ & NS & & $11(4.7)$ & $1.7 \times 10^{-3}$ & 13.6 \\
\hline
\end{tabular}

*Pc: GD without clinical eye phenotype group vs controls.

†PC: proptosis group vs controls.

$¥ P$ : myogenic group vs controls.

Only HLA alleles with corrected $p$ values $<0.05$ are shown. The $p$ value was calculated using $\chi^{2}$ test or Fisher's exact test and corrected for the number of tests performed (Allele $B: 63$, DRB1: 40 , DQA1: 18, DQB1:18; Haplotype A-B-C: 404, DRB1-DQA1-DQB1: 93, DRB1-DQA1-DQB1-DPA1-DPB1: 338, A-B-C-DRB1-DQA1-DQB1-DPA1-DPB1: 1143).

HLA, human leucocyte antigen; NS, not significant; OR, odds ratio; PC, corrected $p$ value.

Table 2 Association of the DRB1*16:02-DQA1 *01:02-DQB1*05:02 haplotype with GD with or without clinical eye disease

\begin{tabular}{|c|c|c|c|c|c|c|c|c|c|c|c|c|}
\hline \multirow[b]{2}{*}{ DRB1 * 16:02 } & \multirow[b]{2}{*}{$\mathrm{DQA1}$ *01:02 } & \multirow[b]{2}{*}{ DQB1*05:02 } & \multirow{2}{*}{$\frac{\text { Controls }}{(2 n=822, \%)}$} & \multicolumn{3}{|c|}{ GD without clinical eye phenotype group } & \multicolumn{3}{|c|}{ Proptosis Group } & \multicolumn{3}{|c|}{ Myogenic group } \\
\hline & & & & $(2 \mathrm{n}=148, \%)$ & $\mathrm{Pc}^{*}$ & OR & $(2 \mathrm{n}=164, \%)$ & Pct & OR & $(2 n=232, \%$ & \%) Pc‡ & OR \\
\hline+ & + & + & $39(4.7)$ & 17 (11.5) & NS & & 27 (16.5) & $3.9 \times 10^{-6}$ & 4.0 & $45(19.0)$ & $3.2 \times 10^{-11}$ & 4.8 \\
\hline- & - & - & $627(76.3)$ & $104(70.3)$ & NS & & $119(72.6)$ & NS & & $146(63.0)$ & $4.6 \times 10^{-3}$ & 0.5 \\
\hline- & + & - & $105(12.8)$ & $15(10.1)$ & NS & & $9(5.5)$ & NS & & $22(9.0)$ & NS & \\
\hline- & - & + & $32(3.9)$ & $6(4.1)$ & NS & & $3(1.8)$ & NS & & $10(4.0)$ & NS & \\
\hline- & + & + & $19(2.3)$ & $6(4.1)$ & NS & & $6(3.7)$ & NS & & $9(4.0)$ & NS & \\
\hline+ & - & - & $0(0)$ & $0(0)$ & NS & & $0(0)$ & NS & & $0(0.0)$ & NS & \\
\hline+ & + & - & $0(0)$ & $0(0)$ & NS & & $0(0)$ & NS & & $0(0.0)$ & NS & \\
\hline+ & - & + & $0(0)$ & $0(0)$ & NS & & $0(0)$ & NS & & $0(0.0)$ & NS & \\
\hline
\end{tabular}

${ }^{*} \mathrm{PC}$ : GD without clinical eye phenotype group vs controls.

†Pc: proptosis group vs controls.

$\ddagger$ Pc: myogenic group vs controls.

The $\mathrm{p}$ value was calculated using $\chi^{2}$ test or Fisher's exact test and corrected for 93 tests performed.

HLA, human leucocyte antigen; NS, not significant; OR, odds ratio; Pc, corrected $p$ value. 
$02^{-} D O A 1 * 01: 02^{-} D Q B 1 * 05: 02^{-}$was a protective factor for the myogenic group, which survived the Bonferroni correction (Pc $<0.05$, OR $=0.53$ ). Our results suggested that HLA-DRB1*16 :02, HLA-DQA1*01:02 and HLA-DQB1*05:02 were associated with the development of clinical GO.

\section{Comparation of OR and linkage disequilibrium}

The OR of significant HLA allele and haplotype between the disease groups and control groups are shown in figure 1, respectively. The ORs of $H L A-D R B 1 * 16: 02$ and HLA$D Q B 1 * 05: 02$ alleles of 'the myogenic group vs control group' showed the top odd (OR: 4.8 and 14.5), followed by 'the proptosis group vs control group' (OR: 4.0 and 10.7) and 'GD without clinical eye phenotype group vs control group' (OR: 2.6 and 9.3), respectively. The ORs of HLA-DRB1*16:02-DQA1*01:02-DQB1*05:02 and HLA$D R B 1$ *16:02-DQA1*01:02-DQB1*05:02-DPA1*02:02-DPB1* 05: 01 haplotypes of 'the myogenic group vs control group' (OR: 4.8 and 6.6) were higher than that in 'the proptosis group vs control group' (OR: 4.0 and 4.0).

Thirty-five alleles had strong LD $\left(\mathrm{r}^{2}>0.33\right.$, 95\% CI 0.70 to 0.98 ) (data not shown). The allele HLA-DRB1*16:02 was linked to HLA-DQA1*01:02 and HLA-DQB1*05:02 tightly $\left(D^{\prime}=1,95 \%\right.$ CI 0.95 to $1.00, \mathrm{r}^{2}=0.62 ; \mathrm{D}^{\prime}=0.99,95 \% \mathrm{CI}$ 0.96 to $\left.1.00, \mathrm{r}^{2}=0.34\right)$.

\section{Computational epitope prediction and binding of TSHR-ECD and IGF-1R-ECD to HLA}

It has been reported that in GO, the pathogenic autoantigenic peptide epitope of TSHR and IGF-1R could be matched to the antigen-binding pocket of HLA molecule and be recognised by $\mathrm{T}$ cell receptors. ${ }^{13}$ We predicted the potential epitopes of THSR-ECD (amino acids 1-418, AA 1-418) and IGF-1R-ECD (AA 1-902), which could be recognised by the HLA-DRA1-DRB1*16:02 and HLA-DQA1*01 :02-DQB1*05:02 heterodimers.

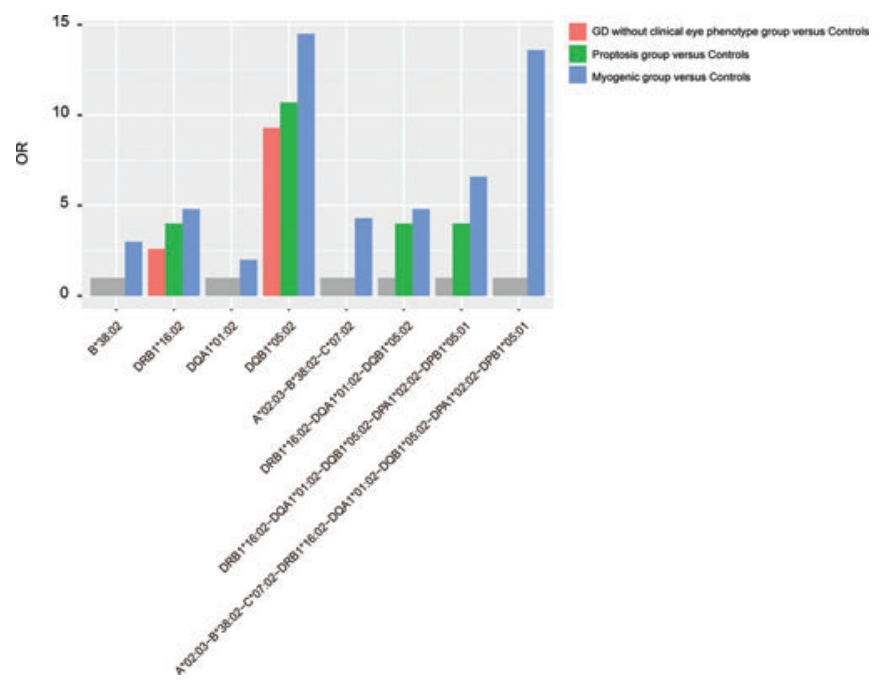

Figure 1 Association analysis OR for significant HLA alleles and haplotypes. The magnitude of OR is indicated by height of column. The OR of 'GD without clinical eye phenotype group vs Controls', 'Proptosis group vs Controls', and 'Myogenic group vs Controls' are shown in pink, blue and green columns, respectively. The grey column represents no significance between two groups.
We identified 1 epitope of THSR-ECD and 3 epitopes of IGF-1R-ECD showing strong binding to HLA-DRA1$D R B 1 * 16: 02$ heterodimer respectively, including 'FLGIFNTGL', 'IRHSHALVS', “ILYIRTNAS' and 'FVFARTMPA'. Similarly, 1 epitope of THSR-ECD and 2 epitopes of IGF-1R-ECD also showing strong binding to HLA-DQA1*01:02-DQB1*05:02 heterodimer respectively, including 'ILEITDNPY', 'NYALVIFEM' and 'NYSFYVLDN' (figure 2). The position of the epitopes with the target sequences 'FLGIFNTGL' and 'ILEITDNPY' were located between AA130-138 and AA155-163 of TSHR-ECD, and 'IRHSHALVS', 'ILYIRTNAS', 'FVFARTMPA', 'NYALVIFEM' and 'NYSFYVLDN' were located between AA390-398, AA601-609, AA820-828, AA114-122, and AA417-425 of IGF-1R-ECD. Furthermore, we predicted the binding affinity between the HLA pocket and epitopes of 'FLGIFNTGL', 'IRHSHALVS', 'ILYIRTNAS', 'FVFARTMPA', 'ILEITDNPY', 'NYALVIFEM' and 'NYSFYVLDN'. The potential sequences 'FLGIFNTGL', 'IRHSHALVS', 'ILYIRTNAS' and 'FVFARTMPA' fitted exactly in the peptide-binding groove between HLA-DRA1-DRB1*16:02 heterodimer, with a docking score $(\Delta G)$ of $-9.1,-9.6,-5.1$ and $-5.9 \mathrm{kcal} /$ mol, respectively (figure 3 ). In addition, the target sequences 'ILEITDNPY', 'NYALVIFEM' and 'NYSFYVLDN' also fitted exactly in the peptide-binding groove between HLA$D Q A 1 * 01: 02-D Q B 1 * 05: 02$ heterodimer. The $\triangle \mathrm{G}$ scores between HLA-DQB1*05:02 and 'ILEITDNPY', 'NYALVIFEM' and 'NYSFYVLDN' were $-6.1,-5.7$ and $-5.9 \mathrm{kcal} / \mathrm{mol}$, respectively (figure 4 ).

\section{DISCUSSION}

In this study, we investigated the association of eight HLA loci with GD and GO in the Southern Chinese population. Our results demonstrated that the alleles $H L A-B * 38: 02,-D R B 1 * 16: 02$, $-D Q A 1 * 01: 02$ and $-D Q B 1 * 05: 02$ and the special haplotypes
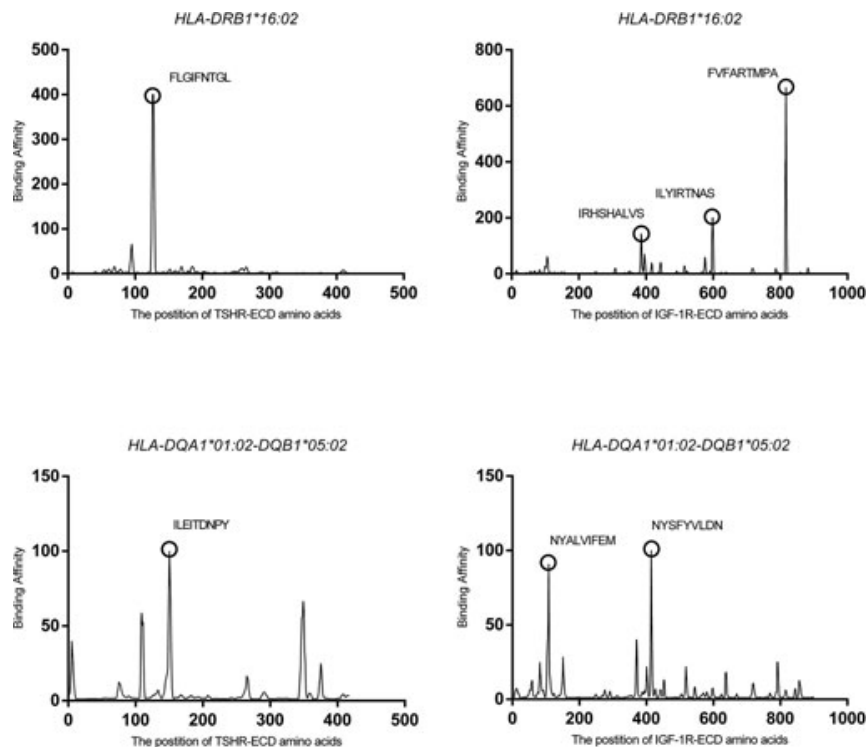

Figure 2 Binding affinity prediction of the TSHR-ECD and IGF-1R-ECD to the HLA-DRB1*16:02 and HLA-DQA1 *01:02-DQB1 *05:02 heterodimers. The NetMHCllpan 3.2 Server was used to predict epitopes within TSHR and IGF-1R protein (TSHR-NP_000360.1 and IGF-1R-NP _000866.1). The $y$-axis presents the binding affinity represented as 1 divided by \%Rank of predicted affinity compared with random natural peptides, while the $\mathrm{x}$-axis represents the position of amino acids. 


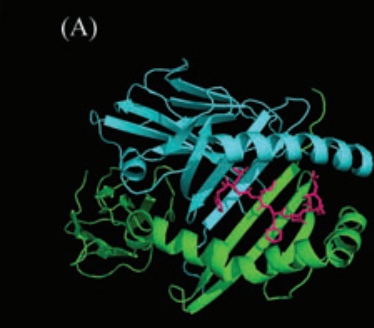

$\Delta \mathrm{G}=-9.1 \mathrm{kcal} / \mathrm{mol}$
(B)

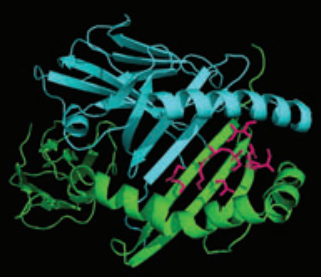

$\Delta \mathrm{G}=-9.6 \mathrm{kcal} / \mathrm{mol}$
(C)

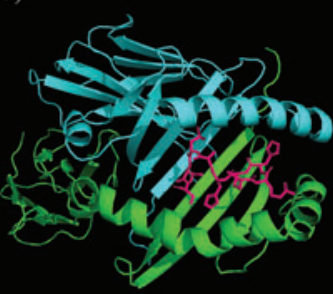

$\Delta \mathrm{G}=-5.1 \mathrm{kcal} / \mathrm{mol}$
(D)

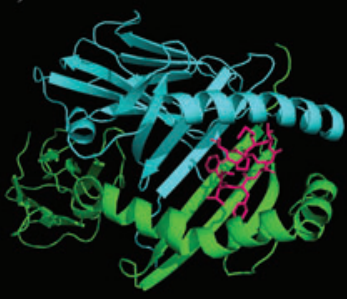

$\Delta \mathrm{G}=-6.9 \mathrm{kcal} / \mathrm{mol}$

Figure 3 Computational docking of TSHR-ECD and IGF-1R-ECD segments to the HLA-DRA1-DRB1 *16:02 heterodimer. (A) 'FLGIFNTGL' of TSHR-ECD binding to the HLA-DRA1-DRB1 *16:02 heterodimer; (B) 'IRHSHALVS' of IGF-1R-ECD binding to the HLA-DRA1-DRB1*16:02 heterodimer; (C) 'ILYIRTNAS' of IGF-1R-ECD binding to the HLA-DRA1-DRB1 *16:02 heterodimer; (D) 'FVFARTMPA' of IGF-1R-ECD binding to the HLA-DRA1-DRB1 *16:02 heterodimer. HLA-DRA1, HLA-DRB1, TSHR-ECD and IGF-1R-ECD segments are shown in blue, green and purple, respectively.

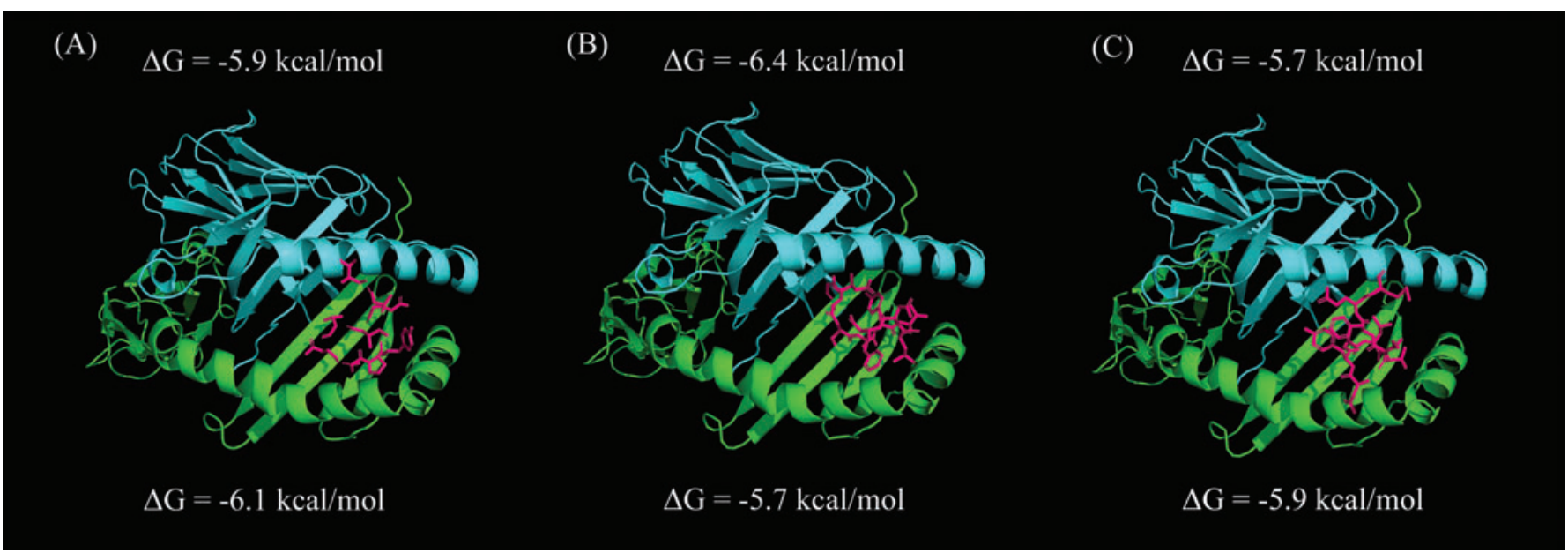

Figure 4 Computational docking of TSHR-ECD and IGF-1R-ECD segments to the HLA-DQA1 *01:02-DQB1 *05:02 heterodimer. (A) 'ILEITDNPY' of TSHR-ECD binding to the HLA-DQA1 *01:02-DQB1 *05:02 heterodimer; (B) 'NYALVIFEM' of IGF-1R-ECD binding to the HLA-DQA1*01:02-DQB1 *05:02 heterodimer; (C) 'NYSFYVLDN' of IGF-1R-ECD binding to the HLA-DQA1 *01:02-DQB1 *05:02 heterodimer. HLA-DQA1, HLA-DQB1, TSHR-ECD and IGF-1R-ECD segments are shown in blue, green and purple, respectively.

formed by these HLA alleles might contribute to the development of different types of ocular phenotypes in GD patients.

The allele frequencies of $H L A-D R B 1 * 16: 02$ and $-D Q B 1 * 05$ :02 in the GD patient in the Southern Chinese population were significantly higher than those in the control group (table 1). Similar findings had been reported in GD patient in Thai, Korea and Han populations in Taiwan. ${ }^{26-28}$ We hypothesised that the alleles HLA-DRB1*16:02 and -DQB1*05:02 might be risk factors in common for GD in the Southern Chinese population or the other Asian population. However, previous associations did not consider different ocular phenotypes in GD. Chen et al revealed that HLA-DRB1*16:02 carried a unique motif of amino acid residues at position 67-74, which might increase the ability to present immunogenic autoantigens. ${ }^{29}$ Alleles HLA-DRB1*16:02 and -DQB1*05:02 were tightly linked in our study due to linkage disequilibrium, which has also been reported in the Asian population. ${ }^{30}$ The combinations of alleles HLA-DRB1*16:02 and -DQB1*05:02 might enhance the susceptibility to some diseases. ${ }^{233031} \mathrm{HLA}$ $D R B 1 * 16$ has been associated with the pathogenesis of the myopathic process of GO in Brazilian patients. ${ }^{7}$ Beside allele $H L A-D R B 1 * 16: 02$, the allele frequencies of $H L A-B * 38: 02$ and $-D Q A 1 * 01: 02$ only in the myogenic group in Southern
Chinese population were significantly higher than those in the control group (table 1). We hypothesised that alleles HLA$B * 38: 02$ and $-D Q A 1 * 01: 02$ might be key risk factors in the development of extraocular-muscle dysfunction in Southern Chinese population with GO.

The HLA haplotype combination of alleles $H L A-B * 38: 02$, $-D R B 1 * 16: 02$, $-D Q A 1 * 01: 02$ and $-D Q B 1 * 05: 02$ might contribute to the development of ocular phenotype in GD patients. Firstly, the haplotypes HLA-DRB1*16:02-DQA1*01:02DQB1*05:02 and HLA-DRB1*16:02-DQA1*01:02-DQB1*05 :02-DPA1*02:02-DPB1*05:01 might be common risk factors for the proptosis and myogenic phenotypes of GO in Southern Chinese population (table 1). Secondly, the frequencies of class I haplotype $H L A-A * 02: 03-B * 38: 02-C^{*} 07: 02$ and eight HLA loci haplotype HLA-A*02:03-B*38:02-C*07:02-DRB1*16:02$D Q A 1 * 01: 02-D Q B 1 * 05: 02-D P A 1 * 02: 02-D P B 1 * 05: 01$ in the myogenic group were significantly higher than those in the control group (table 1). Interestingly, the frequency of the haplotype lacking of alleles HLA-DRB1*16:02, -DQA1*01:02 and $D Q B 1 * 05: 02$ in the myogenic group was significantly lower than that in the control group (table 2). Lastly, the ORs of the significant HLA alleles and haplotypes in myogenic group were higher than those in the proptosis and GD without clinical eye 
phenotype group respectively (table 1 , figure 1 ). Therefore, the combination of alleles HLA-B*38:02, -DRB1*16:02, $-D Q A 1 * 01$ : 02 and $-D Q B 1 * 05: 02$ might contribute to different phenotypes of $\mathrm{GO}$ with different degrees.

Notably, some disease-causing autoantigenic peptides were only fitted into the peptide- binding pockets of specific kind of HLA-DRA1-DRB1 and HLA-DQA1-DQB1 heterodimers, and recognised by the $\mathrm{CD} 4^{+}$T-cell receptor. ${ }^{13}{ }^{15-18} 2332$ Some previous studies showed that the alleles HLA-DRB1*16:02 and/or HLA-DQB1*05:02 might be mediated by autoantibodies in GD, anti-NMDAR encephalitis and anti-IFN- $\gamma$ disseminated nontuberculous mycobacterial infections myasthenia gravis, and juvenile ocular myasthenia gravis. ${ }^{23} 2930$ Shu et al found that the epitope 'FRAITSTLA' of autoantibody to the NR1 subunit of NMDAR binds to HLA-DRA1-DRB1*16:02 heterodimer on the surface of APCs, which might provoke the pathogenesis of antiNMDAR encephalitis. ${ }^{23}$ Our study also discovered the association between heterodimers (HLA-DRA1-DRB1*16:02 and HLA$D Q A * 01: 02-D Q B 1 * 05: 02$ ) and the antigens (TSHR and IGF$1 \mathrm{R})$. Interestingly, bioinformatic analyses suggested that some epitopes ('FLGIFNTGL' of TSHR-ECD, 'IRHSHALVS', 'ILYIRTNAS' and 'FVFARTMPA' of IGF-1R-ECD) strongly bind to the HLA-DR $\alpha$-DR $\beta 1$ pocket groove, encoded by the HLA-DRA1-DRB1*16:02 heterodimer. Simultaneously, the HLA-DQA*01:02-DQB1*05:02 heterodimer encoded the pocket groove of HLA-DQ $\alpha 1-\mathrm{DQ} \beta 1$ peptide-binding groove combined with the potential epitopes ('ILEITDNPY' of THSRECD, 'NYALVIFEM' and 'NYSFYVLDN' of IGF-1R-ECD), strongly. These peptides were fitted tightly in the peptidebinding groove of HLA-DR $\alpha$-DR $\beta 1$ or HLA-DQ $\alpha 1-D Q \beta 1$ with relatively lower docking scores (especial peptides, 'FLGIFNTGL' of TSHR-ECD and 'IRHSHALVS' of IGF-1R-ECD). Previous studies have found that the epitopes ('FLGIFNTGL' and (ILEITDNPY') of THSR-ECD might be the immunodominant T-cell epitope that binds to the HLA-DR3 molecule in silico and in vitro. ${ }^{16-18} 333^{34}$ These epitopes of TSHR-ECD or IGF-1R-ECD might favour binding to the pocket groove of special heterodimers (HLA-DR $\alpha$-DR $\beta 1$ or HLA-DQ $\alpha$-DQ $\beta 1$ ), increasing the capacity in APCs, to present the disease-causing autoantigenic peptides to the T-cell receptors, which might predispose to GD with and without clinical GO in immunogenicity.

We firstly conducted high-resolution genotyping for eight HLA loci in 272 unrelated GDpatient with and without clinical GO in Southern Chinese at both allelic andhaplotypic levels. These results showed that the specific HLA alleles andhaplotypes of GO were prevalent in patients with GD or GO in Southern Chinese, which is a different region and ethnic group from previous studies. Inaddition, we found that the HLA alleles and haplotypes contributed to differentprogression of GO. Furthermore, we performed an in-silico analysis for the bindingaffinities between HLA class II and peptides from the two most extensivelystudied autoantigens primarily. Our results indicated that different HLAalleles and haplotypes could be used as biomarkers and predict the progressionof different phenotypes of GO in Southern Chinese. However, there were some limitations in this study. The number of patients with GD and GO was relatively small, and the participation seems to be populationspecific, which might have limited our results generalising to the general population. We found the female frequency of the patients was significantly higher than those in the control group. However, the sex mismatch might not impact the HLAcontribution to GD since the inheritance of HLA loci is autosomal dominant. We should have proven that the binding condition might provoke the progression to GO between the potential peptides of THSR or IGF-1R and HLA-DRA1$D R B 1 * 16: 02$ or HLA-DQA*01:02-DQB1*05:02 heterodimers in vitro and in vivo.

\section{CONCLUSIONS}

Taken together, the current study suggested that alleles HLA$B * 38: 02,-D R B 1 * 16: 02,-D Q A 1 * 01: 02$ and $-D Q B 1 * 05: 02$ and corresponding haplotypes might contribute to the development of GD, proptosis and myogenic phenotypes of GO in the Southern Chinese population. In addition, we predicted that some potential critical epitopes of TSHR-ECD or IGF-1R-ECD favoured binding to HLA-DRA1-DRB1*16:02 and HLA$D Q A * 01: 02-D Q B 1 * 05: 02$ heterodimers tightly, by bioinformatic analysis. These findings indicated that the associations of alleles HLA-B*38:02, -DRB1*16:02, -DQA1*01:02 and $D Q B 1 * 05: 02$ and corresponding haplotypes with the development of GD and clinical GO might be due to affecting the affinity between HLA peptide-binding groove and antigenic peptides of TSHR-ECD and IGF-1R-ECD.

\section{Author affiliations}

${ }^{1}$ Shenzhen Eye Institute, Shenzhen Eye Hospital Affiliated to Jinan University, Shenzhen, Guangdong, China

${ }^{2}$ School of Ophthalmology \& Optometry, Shenzhen University, Shenzhen, Guangdong, China

${ }^{3}$ Cancer Center, Wuzhou Red Cross Hospital, Wuzhou, Guangxi, China

${ }^{4}$ Immunogenetics Laboratory, Shenzhen Blood Center, Shenzhen, Guangdong, China

${ }^{5}$ Department of Transfusion Medicine, School of Laboratory Medicine and Biotechnology, Southern Medical University, Guangzhou, China

Acknowledgements We are indebted to the participants for their excellent cooperation and support. We would like to thank the volunteer blood donors for generously providing blood samples for this study.

Contributors $\mathrm{XH}$ and $\mathrm{GL}$ contributed equally to this work. ZD and JZ contributed equally to this work. XH, JZ and ZD contributed to study design, data analysis, result interpretation and manuscript drafting. GL contributed to provide the information and blood samples of participates. XH, SM, JC, JR, TZ, WC, SP, YW and YY contributed to data acquisition and management. XH, MT, TZ and JZ contributed to data analysis. Al authors contributed to manuscript drafting and its critical revision for final content.

Funding This study was supported by Science, Technology and Innovation Commission of Shenzhen Municipality under Grant (number GJHZ20180420180937076, number JCYJ20180228164400218 and number JCYJ20190806152001762), Sanming Project of Medicine in Shenzhen Grant (number SZSM201812090), and Shenzhen Key Medical Discipline Construction Fund (number SZXK070).

Competing interests None declared.

\section{Patient consent for publication Not require.}

Provenance and peer review Not commissioned; externally peer reviewed.

Data availabilty statement Data are available upon reasonable request.

Open access This is an open access article distributed in accordance with the Creative Commons Attribution Non Commercial (CC BY-NC 4.0) license, which permits others to distribute, remix, adapt, build upon this work non-commercially, and license their derivative works on different terms, provided the original work is properly cited, appropriate credit is given, any changes made indicated, and the use is noncommercial. See: http://creativecommons.org/licenses/by-nc/4.0/.

\section{ORCID iDs}

Xiaosheng Huang http://orcid.org/0000-0002-6375-0816

Zhihui Deng http://orcid.org/0000-0002-2046-1467

Jun Zhao http://orcid.org/0000-0002-7285-5812

\section{REFERENCES}

1 Bahn RS. Graves' ophthalmopathy. N Engl J Med 2010;362:726-38.

2 Bednarczuk T, Hiromatsu Y, Fukutani T, et al. Association of cytotoxic T-lymphocyteassociated antigen-4 (CTLA-4) gene polymorphism and non-genetic factors with Graves' ophthalmopathy in European and Japanese populations. Euro J Endocrinol 2003:148:13-18.

3 Chu X, Yang M, Song ZJ, et al. Fine mapping MHC associations in Graves' disease and its clinical subtypes in Han Chinese. J Med Genet 2018;55:685-92. 
4 Okada Y, Momozawa Y, Ashikawa K, et al. Construction of a population-specific HLA imputation reference panel and its application to Graves' disease risk in Japanese. Nat Genet 2015;47:798-802.

5 Dendrou CA, Petersen J, Rossjohn J, et al. HLA variation and disease. Nat Rev Immunol 2018;18:325-39.

6 Khalilzadeh 0, Noshad S, Rashidi A, et al. Graves' ophthalmopathy: a review of immunogenetics. Curr Genomics 2011;12:564-75.

7 Akaishi PM, Cruz AA, Silva FL, et al. The role of major histocompatibility complex alleles in the susceptibility of Brazilian patients to develop the myogenic type of Graves' orbitopathy. Thyroid 2008;18:443-7.

8 Bednarczuk T, Hiromatsu Y, Seki N, et al. Association of tumor necrosis factor and human leukocyte antigen DRB1 alleles with Graves' ophthalmopathy. Hum Immunol 2004;65:632-9.

9 Ohtsuka K, Nakamura Y. Human leukocyte antigens associated with hyperthyroid Graves ophthalmology in Japanese patients. Am J Ophthalmol 1998;126:805-10.

10 Kendall-Taylor P, Stephenson A, Stratton A, et al. Differentiation of autoimmune ophthalmopathy from Graves' hyperthyroidism by analysis of genetic markers. Clin Endocrinol (Oxf) 1988;28:601-10.

11 Weetman A, Zhang L, Webb S, et al. Analysis of HLA-DQB and HLA-DPB alleles in Graves' disease by oligonucleotide probing of enzymatically amplified DNA. Clin Endocrinol (Oxf) 1990;33:65-71.

12 Frecker M, Stenszky V, Balazs C, et al. Genetic factors in Graves' ophthalmopathy. Clin Endocrinol (Oxf) 1986;25:479-85.

13 Wang Y, Smith TJ. Current concepts in the molecular pathogenesis of thyroid-associated ophthalmopathy. Invest Ophthalmol Vis Sci 2014;55:1735-48.

14 Smith TJ. Recognizing the putative role for TSH receptor expressing fibrocytes in thyroid-associated ophthalmopathy may solve several mysteries. Nat Rev Endocrinol 2015:11:171-81.

15 Tsui S, Naik V, Hoa N, et al. Evidence for an association between thyroid-stimulating hormone and insulin-like growth factor 1 receptors: a tale of two antigens implicated in Graves' disease. J Immunol 2008;181:4397-405.

16 Inaba H, Pan D, Shin YH, et al. Immune response of mice transgenic for human histocompatibility leukocyte Antigen-DR to human thyrotropin receptor-extracellular domain. Thyroid 2009;19:1271-80.

17 Inaba H, Martin W, Ardito $M$, et al. The role of glutamic or aspartic acid in position four of the epitope binding motif and thyrotropin receptor-extracellular domain epitope selection in Graves' disease. J Clin Endocrinol Metab 2010;95:2909-16.

18 Inaba H, De Groot LJ, Akamizu T. Thyrotropin receptor epitope and human leukocyte antigen in Graves' disease. Front Endocrinol (Lausanne) 2016:7:120.
19 Krieger CC, Perry JD, Morgan SJ, et al. TSH/IGF-1 receptor cross-talk rapidly activates extracellular signal-regulated kinases in multiple cell types. Endocrinology 2017;158:3676-83.

20 Mahdavi M, Moreau V, Kheirollahi M. Identification of B and T cell epitope based peptide vaccine from IGF-1 receptor in breast cancer. J Mol Graph Model 2017:75:316-21.

21 Voorter CE, van den Berg-loonen EM. Sequence-based typing of the complete coding sequence of DQA1 and phenotype frequencies in the Dutch Caucasian population. Hum Immunol 2006;67:756-63.

22 Jensen KK, Andreatta M, Marcatili P, et al. Improved methods for predicting peptide binding affinity to MHC class II molecules. Immunology 2018;154:394-406.

23 Shu Y, Qiu W, Zheng J, et al. HLA class II allele DRB 1*16:02 is associated with antiNMDAR encephalitis. J Neurol Neurosurg Psychiatry 2019;90:652-8.

24 Excoffier $\mathrm{L}$, Lischer HE. Arlequin suite ver 3.5: a new series of programs to perform population genetics analyses under linux and windows. Mol Ecol Resour 2010;10:564-7.

25 Lewontin RC. On measures of gametic disequilibrium. Genetics 1988;120:849-52

26 Wongsurawat T, Nakkuntod J, Charoenwongse P, et al. The association between HLA class II haplotype with Graves' disease in Thai population. Tissue Antigens 2006;67:79-83.

27 Park MH, Park YJ, Song EY, et al. Association of HLA-DR and -DQ genes with Graves disease in Koreans. Hum Immunol 2005;66:741-7.

28 Chen PL, Fann CS, Chu CC, et al. Comprehensive genotyping in two homogeneous Graves' disease samples reveals major and novel HLA association alleles. PloS One 2011;6:e16635.

29 Chen Y, Li S, Huang R, et al. Comprehensive meta-analysis reveals an association of the HLA-DRB1 ${ }^{*} 1602$ allele with autoimmune diseases mediated predominantly by autoantibodies. Autoimmun Rev 2020;19:102532.

30 Chi CY, Chu CC, Liu JP, et al. Anti-IFN- $\gamma$ autoantibodies in adults with disseminated nontuberculous mycobacterial infections are associated with HLA-DRB1 *16:02 and HLA-DQB $1{ }^{*} 05: 02$ and the reactivation of latent varicella-zoster virus infection. Blood 2013:121:1357-66.

31 Terao C, Yoshifuji H, Yamano Y, et al. Genotyping of relapsing polychondritis identified novel susceptibility HLA alleles and distinct genetic characteristics from other rheumatic diseases. Rheumatology (Oxford) 2016;55:1686-92.

32 Kim TJ, Lee ST, Moon J, et al. Anti-LGI1 encephalitis is associated with unique HLA subtypes. Ann Neurol 2017;81:183-92.

33 Inaba H, Moise L, Martin W, et al. Epitope recognition in HLA-DR3 transgenic mice immunized to TSH-R protein or peptides. Endocrinology 2013;154:2234-43.

34 Sawai Y, DeGroot LJ. Binding of human thyrotropin receptor peptides to a Graves' disease-predisposing human leukocyte antigen class II molecule. J Clin Endocrinol Metab 2000:85:1176-9. 